\title{
Effect of various doses of vitamin D supplementation on pregnant women with gestational diabetes mellitus: A randomized controlled trial
}

\author{
QINGYING ZHANG ${ }^{1,2}$, YAN CHENG ${ }^{1}$, MULAN HE $^{1}$, TINGTING LI ${ }^{1}$, ZIWEN MA ${ }^{1}$ and HAIDONG CHENG ${ }^{1,2}$ \\ ${ }^{1}$ Department of Obstetrics and ${ }^{2}$ Shanghai Key Laboratory of Female Reproductive Endocrine Related Diseases, \\ Obstetrics and Gynecology Hospital of Fudan University, Shanghai 200090, P.R. China
}

Received February 26, 2015; Accepted November 25, 2015

DOI: $10.3892 /$ etm.2016.3515

\begin{abstract}
It has previously been reported that the influence of vitamin D on the metabolism of calcium and phosphorus is associated with diabetes, cardiovascular disease, Alzheimer's disease, cancer and other systemic diseases, and is considered an important indicator of general health. The present study was conducted to determine the effect of various doses of vitamin D supplementation on glucose metabolism, lipid concentrations, inflammation and the levels of oxidative stress of pregnant women with gestational diabetes mellitus (GDM). The present randomized, double-blind placebo-controlled clinical trial was conducted on 133 pregnant women with GDM during weeks 24-28 of pregnancy. The patients were randomly divided into four groups. The control group $(n=20)$ received a placebo (sucrose; one granule/day), the low dosage group $(n=38)$ received the daily recommended intake of 200 IU vitamin D (calciferol) daily, the medium dosage group $(n=38)$ received 50,000 IU monthly $(2,000$ IU daily for 25 days) and the high dosage group $(n=37)$ received 50,000 IU every 2 weeks (4,000 IU daily for 12.5 days). The general characteristics and dietary intakes of the patients with GDM were similar between each group. Using ELISA kits, it was determined that insulin, homeostatic model assessment-insulin resistance and total cholesterol were significantly reduced by high dosage vitamin $\mathrm{D}$ supplementation $(\mathrm{P}<0.05)$. Total antioxidant capacity and total glutathione levels were significantly elevated as a result of high dosage vitamin D supplementation $(\mathrm{P}<0.01)$. In conclusion, high-dose vitamin $\mathrm{D}$ supplementation (50,000 IU every 2 weeks) significantly improved insulin resistance in pregnant women with GDM.
\end{abstract}

Correspondence to: Dr Haidong Cheng, Department of Obstetrics, Obstetrics and Gynecology Hospital of Fudan University, 128 Shenyang Road, Yangpu, Shanghai 200090, P.R. China E-mail: haidongchengMD@163.com

Key words: vitamin D supplementation, pregnant women, gestational diabetes mellitus, randomized controlled trial

\section{Introduction}

Gestational diabetes mellitus (GDM) is defined as an abnormal glucose metabolism that initially occurs or is first recognized during the pregnancy period, and is a relatively common complication of pregnancy in China (1). In recent years, with the improvement of medical diagnostic technology and the living standards of the human population, GDM incidence has increased $(2,3)$. Patients with GDM are prone to pregnancy-induced hypertension, polyhydramnios, infections, ketoacidosis and other complications; if the control of blood glucose levels remains unfavorable for a prolonged duration of time, it may result in chronic intrauterine fetal hypoxia, growth abnormalities, malformations, neonatal hyperbilirubinemia and hypoglycemia respiratory distress syndrome, amongst others $(4,5)$.

Previous studies have revealed that GDM may increase fetal growth restriction, fetal distress, preterm delivery and polyhydramnios during pregnancy, in addition to an increase in the risk of type 2 diabetes and the incidence of cesarean pain $(6,7)$. Furthermore, GDM may result in adverse effects in the offspring, including fetal macrosomia, defects associated with premature birth, low birth weight, neonatal respiratory distress syndrome and neonatal jaundice (8). Additional research has revealed that the aforementioned adverse pregnancy outcomes are associated with maternal blood glucose levels; thus the effective control of blood sugar levels in pregnant women has the ability to successfully reduce adverse pregnancy outcomes (9). Therefore, the primary goal for successful medical management of GDM is to maintain the concentration of maternal blood glucose, particularly postprandial blood glucose levels, within the ideal range (10). It has been hypothesized that the mechanism of GDM involves an increase in the hormones produced by the placenta known to resist insulin, which results in a decreased sensitivity to insulin in pregnant women $(11,12)$. This consolidates the scientific basis of medical nutrition therapy for the treatment of GDM (13). Medical nutrition therapy aims to treat high blood sugar through scientific dietary adjustments in combination with exercise and blood glucose level monitoring. The American Diabetes Association recommends that all patients be evaluated for GDM risk according to their food intake, 
metabolic status and lifestyle by a professional nutritionist, who can develop an individualized medical nutrition therapy according to the patients height, weight and gestational age (14). As the primary method for the treatment of GDM, medical nutrition therapy has gained increasing clinical attention.

Vitamin D is a fat-soluble steroid hormone. Recent studies have indicated its involvement in cardiovascular disease, cancer, autoimmune diseases and diabetes. This is an effect of the widespread distribution of its receptor, vitamin $\mathrm{D}$ receptor (VDR), which interacts with the active form of vitamin $\mathrm{D}$, 1,25-dihydroxyvitamin $\mathrm{D}_{3}$, in vivo, in addition to the classic role of VDR in the regulation of calcium and phosphate metabolism (15). VDR is also present in islet $\beta$ cells. As vitamin $\mathrm{D}$ affects insulin secretion and function by acting on the VDR of $\beta$ cells, it has become a novel hotspot of diabetes research. Numerous studies have confirmed that vitamin D is associated with diabetes in patients that are not pregnant, and further investigation to determine its mechanism of action has been conducted $(16,17)$.

The present study evaluated the effect of different doses of vitamin D supplementation on glucose metabolism, lipid concentrations, inflammation and the oxidative stress levels of pregnant women with GDM, in order to assess whether vitamin D may improve the treatment of GDM.

\section{Patients and methods}

Participants. In the present randomized, double-blind, controlled clinical trial, 283 pregnant women were recruited from the Obstetrics and Gynecology Hospital of Fudan University (Shanghai, China), between September 2009 and November 2014 (Fig. 1). The exclusion criteria included women with: Diabetes or GDM treated with insulin, thyroid or parathyroid disorders, polycystic ovary disease prior to pregnancy, a body mass index (BMI) of $>30 \mathrm{~kg} / \mathrm{m}^{2}$ prior to pregnancy and women who had received vitamin $\mathrm{D}$ supplementation in the 6 months that preceded the trial. In addition, a GDM diagnosis of $<12$ weeks was required for eligibility. To detect a minimum of $7 \mathrm{ng} / \mathrm{ml}$ difference in the mean of vitamin D between groups with $80 \%$ power, a standard deviation of 10.5 was assumed, as determined in previous studies $(18,19)$, and $\alpha=0.05$ was used. The present study therefore aimed to recruit 133 patients to allow for a $10 \%$ dropout throughout the duration of the study.

Study design. All patients provided written informed consent for participation in the present study, which was approved by the Institutional Ethics Committee [approval no. (2009)21]. During the initial antenatal visit between 24 and 28 weeks of pregnancy, a blood sample was obtained to measure fasting blood sugar (FBS), insulin, 25-hydroxyvitamin D [25(OH)D] and calcium levels. The patients $(n=133)$ were then randomly divided into four groups (Fig. 1). Computer-generated random number lists were produced by an independent researcher. Patients and researchers were blind to treatment assignment. All dosing was performed via the oral route. The control group $(n=20)$ received a placebo (sucrose; one granule/day), the low dosage group $(n=38)$ received the daily recommended intake of 200 IU vitamin D (calciferol; Costco Wholesale
Corporation, Issaquah, WA, USA) daily, the medium dosage group $(n=38)$ received 50,000 IU monthly $(2,000$ IU daily for 25 days $)$ and the high dosage group $(n=37)$ received 50,000 IU every 2 weeks (4,000 IU daily for 12.5 days). Supplementation commenced at 24-28 weeks of pregnancy and continued until delivery. Patients received a follow-up examination every month during pregnancy and were evaluated for indications of adverse effects to vitamin D, including headaches and vomiting. A blood sample for the measurement of fasting plasma glucose (FPG), insulin, vitamin D and calcium levels was retrieved from each participant at the end of pregnancy.

Assessment of variables. Height and prepregnancy weight were obtained from the records of the patients that existed within the clinic. A midwife at the maternity clinic performed anthropometric measurements at the study baseline and 9 weeks subsequent to the intervention. The height of patients was measured to the nearest $0.1 \mathrm{~cm}$ with a nonstretched tape measure (Seca GmbH \& Co. KG, Hamburg, Germany). The body weight of pregnant women was measured to the nearest $0.1 \mathrm{~kg}$ following overnight fasting and wearing minimal clothing, by the use of a digital scale (Seca GmbH \& Co. KG). BMI was determined as weight $(\mathrm{kg}) /$ height $\left(\mathrm{m}^{2}\right)$. Venous blood samples were harvested and immediately centrifuged at $10,000 \mathrm{x}$ g for $15 \mathrm{~min}$ (Wuxi Ruijiang Analysis Instrument Co., Ltd., Wuxi, China) to separate the serum. Serum samples were then stored at $-80^{\circ} \mathrm{C}$ prior to analysis.

Serum 25(OH)D concentrations were assayed using a commercial ELISA kit (H191; Jiancheng Bioengineering Institute, Nanjing, China). Vitamin D sufficiency was defined as $>30 \mathrm{ng} / \mathrm{ml} 25(\mathrm{OH}) \mathrm{D}$, insufficiency as $25(\mathrm{OH}) \mathrm{D}$ levels of 20-30 ng/ml and deficiency as serum 25(OH)D levels of $<20 \mathrm{ng} / \mathrm{ml}$. Serum calcium (C004-2), phosphorus, (C006) magnesium (C005), zinc (E011; all Jiancheng Bioengineering Institute) and selenium (E-EL-H2065c; Elabscience Bioengineering Co., Ltd., Wuhan, China) concentrations were also assayed using commercial ELISA kits. FPG (R057), serum insulin (H203), insulin resistance (R056) [as determined by homeostatic model assessment-insulin resistance (HOMA-IR)], total cholesterol change (F002-1), triglyceride levels (A110-1), high-sensitivity C-reactive protein (hs-CRP; E024), plasma total antioxidant capacity (TAC; A015) and glutathione (GSH; A005) changes were measured with commercial ELISA kits purchased from Beyotime Institute of Biotechnology (Haimen, China).

Statistical analysis. To ensure the normal distribution of variables, the Kolmogorov-Smirnov test was applied. All statistical analyses were performed using SPSS version 17.0 (SPSS, Inc., Chicago, IL, USA). Data are presented as means \pm standard deviation (SD). Non-parametric tests were employed to compare the groups, and to compare baseline and endpoint measurements. For comparisons within groups, the Wilcoxon test was used. $\mathrm{P}<0.05$ was considered to indicate a statistically significant difference.

\section{Results}

General characteristics of pregnant women with GDM. In the present randomized controlled trial, 37 cases from the 
Table I. General characteristics of patients with GDM.

\begin{tabular}{|c|c|c|c|c|c|}
\hline Characteristic & $\begin{array}{l}\text { Control } \\
(n=20)\end{array}$ & $\begin{array}{l}\text { Low dosage } \\
\qquad(n=38)\end{array}$ & $\begin{array}{l}\text { Medium dosage } \\
\qquad(\mathrm{n}=38)\end{array}$ & $\begin{array}{l}\text { High dosage } \\
\qquad(n=37)\end{array}$ & P-value \\
\hline Age, year & $29.8 \pm 4.7$ & $30.3 \pm 5.1$ & $29.4 \pm 4.9$ & $30.1 \pm 4.5$ & 0.87 \\
\hline Height, cm & $160.1 \pm 5.1$ & $158 \pm 5.7$ & $157.8 \pm 6.6$ & $159.1 \pm 5.1$ & 0.62 \\
\hline Prepregnancy weight, kg & $70.5 \pm 9.4$ & $69.1 \pm 8.9$ & $69.7 \pm 8.8$ & $70.1 \pm 9.2$ & 0.71 \\
\hline Weight at study baseline, $\mathrm{kg}$ & $79.1 \pm 10.1$ & $79.6 \pm 10.6$ & $78.2 \pm 9.9$ & $78.8 \pm 12.1$ & 0.76 \\
\hline Weight at end of trial, $\mathrm{kg}$ & $81.8 \pm 11.8$ & $81.1 \pm 11.2$ & $80.4 \pm 12.5$ & $80.9 \pm 10.3$ & 0.87 \\
\hline Prepregnancy BMI, kg/m² & $27.1 \pm 3.6$ & $27.4 \pm 4.6$ & $26.9 \pm 4.1$ & $27.2 \pm 4.3$ & 0.89 \\
\hline BMI at study baseline, $\mathrm{kg} / \mathrm{m}^{2}$ & $31.1 \pm 3.9$ & $30.6 \pm 4.1$ & $31.1 \pm 3.9$ & $30.6 \pm 4.1$ & 0.63 \\
\hline $\mathrm{BMI}$ at end of trial, $\mathrm{kg} / \mathrm{m}^{2}$ & $32.3 \pm 4.3$ & $31.1 \pm 5.2$ & $32.8 \pm 3.8$ & $31.8 \pm 4.4$ & 0.87 \\
\hline
\end{tabular}

GDM, gestational diabetes mellitus; BMI, body mass index.

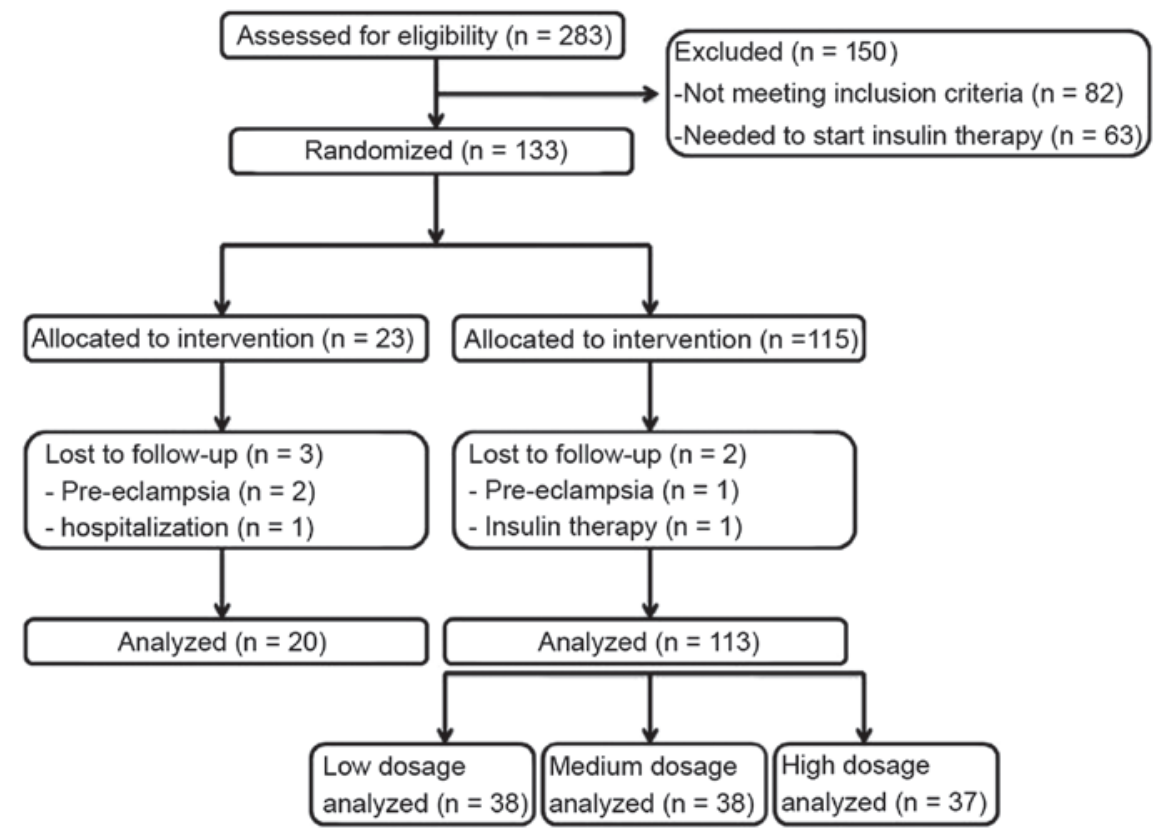

Figure 1. Flow diagram summarizing patient progress through the trial.

high dosage group, 38 cases from the medium dosage group, 38 cases from the low dosage group and 20 pregnant women from the control group completed participation (Fig. 1). Table I displays the general characteristics of the pregnant female patients with GDM in each of the groups prior to intervention. In the control group, the mean age $\pm \mathrm{SD}$ was $29.8 \pm 4.7$ years, the mean height $\pm \mathrm{SD}$ was $160.1 \pm 5.1 \mathrm{~cm}$, the mean prepregnancy weight $\pm \mathrm{SD}$ was $70.5 \pm 9.4 \mathrm{~kg}$ and the mean prepregnancy $\mathrm{BMI} \pm \mathrm{SD}$ was $27.1 \pm 3.6 \mathrm{~kg} / \mathrm{m}^{2}$. Prior to intervention, the mean age, height, FBS, prepregnancy weight and prepregnancy BMI were not significantly different between the three groups $(\mathrm{P}>0.05)$.

Dietary intakes of pregnant women with GDM. Dietary intakes of patients with GDM are displayed in Table II, as determined by ELISA of serum blood samples. The mean energy, carbohydrate, protein, dietary fiber, vitamin D, calcium, phosphorus, magnesium, zinc and selenium levels in the control group were similar to those in each of the treatment groups $(\mathrm{P}>0.05)$.

High and medium doses of vitamin D supplementation reduces insulin and HOMA-IR levels in patients with GDM. Fig. 2 revealed that FPG levels were not significantly affected by any of the low, medium and high vitamin D supplementation groups (96.12, 88.59 and $84.73 \mathrm{mg} / \mathrm{dl}$, respectively), as compared with the control group (92.49 mg/dl) (Fig. 2A; P>0.05). Conversely, as compared with the control group, the high and medium vitamin D supplementation groups effectually reduced insulin (Fig. 2B; 9.21 vs. 5.01 and $4.2 \mathrm{IU} / \mathrm{ml}$, respectively) and HOMA-IR (Fig. 2C; 2.87 vs. 1.52 and 1.18, respectively) concentration levels in patients with GDM $(\mathrm{P}<0.01)$.

High and medium doses of vitamin D supplementation decreases total cholesterol in patients with GDMs. Fig. 3A revealed that the medium and high vitamin D supplementation 
Table II. Dietary intakes of pregnant women with GDM.

\begin{tabular}{|c|c|c|c|c|c|}
\hline Dietary intake & $\begin{array}{l}\text { Control } \\
(\mathrm{n}=20)\end{array}$ & $\begin{array}{l}\text { Low dosage } \\
\qquad(\mathrm{n}=38)\end{array}$ & $\begin{array}{l}\text { Medium dosage } \\
\quad(\mathrm{n}=38)\end{array}$ & $\begin{array}{l}\text { High dosage } \\
\qquad(\mathrm{n}=37)\end{array}$ & P-value \\
\hline Energy, kcal/day & $2,411 \pm 189$ & $2,392 \pm 211$ & $2,388 \pm 198$ & $2,396 \pm 192$ & 0.75 \\
\hline Carbohydrate, g/day & $329 \pm 51$ & $338 \pm 44$ & $333 \pm 48$ & $335 \pm 46$ & 0.82 \\
\hline Protein, g/day & $84 \pm 17$ & $86 \pm 16$ & $81 \pm 19$ & $83 \pm 18$ & 0.36 \\
\hline Dietary fiber, g/day & $19.5 \pm 4.6$ & $20.1 \pm 4.6$ & $19.7 \pm 4.4$ & $20.7 \pm 4.1$ & 0.24 \\
\hline Vitamin D, mg/day & $2.9 \pm 0.5$ & $2.8 \pm 0.9$ & $2.9 \pm 0.6$ & $2.8 \pm 0.7$ & 0.37 \\
\hline Calcium, g/day & $1.15 \pm 0.17$ & $1.15 \pm 0.14$ & $1.15 \pm 0.16$ & $1.15 \pm 0.15$ & 0.91 \\
\hline Phosphorus, g/day & $1.18 \pm 0.22$ & $1.22 \pm 0.12$ & $1.20 \pm 0.16$ & $1.19 \pm 0.19$ & 0.76 \\
\hline Magnesium, mg/day & $296 \pm 73$ & $294 \pm 66$ & $292 \pm 71$ & $291 \pm 69$ & 0.66 \\
\hline Zinc, mg/day & $9.3 \pm 2.7$ & $9.0 \pm 2.4$ & $9.1 \pm 2.1$ & $8.9 \pm 2.7$ & 0.79 \\
\hline Selenium, mg/day & $118 \pm 31.9$ & $116 \pm 29.5$ & $123 \pm 29.1$ & $121 \pm 28.9$ & 0.39 \\
\hline
\end{tabular}

GDM, gestational diabetes mellitus.

A

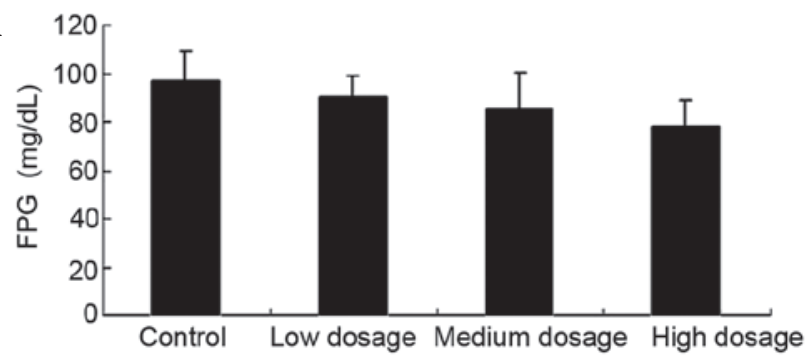

C
B

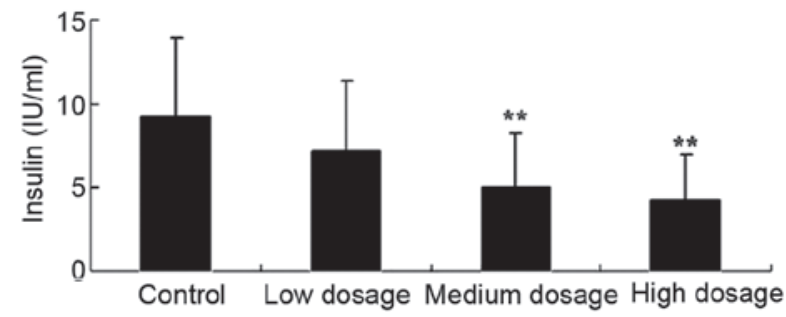

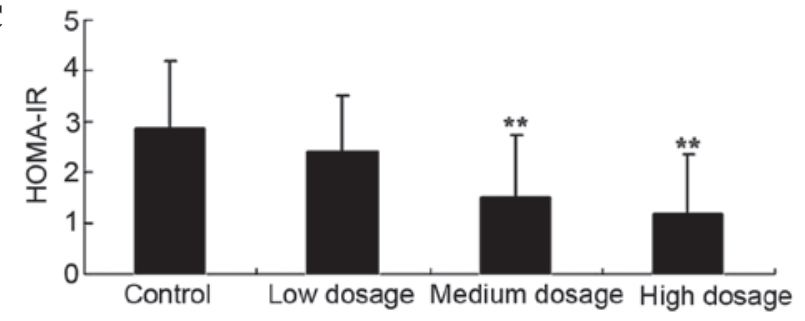

Figure 2. Effect of different doses of vitamin D supplementation on (A) FPG (B) insulin and (C) HOMA-IR in patients with gestational diabetes mellitus. Data are presented as mean \pm standard deviation. ${ }^{* *} \mathrm{P}<0.01$ vs. control group. FPG, fasting plasma glucose; HOMA-IR, homeostatic model assessment-insulin resistance.

A

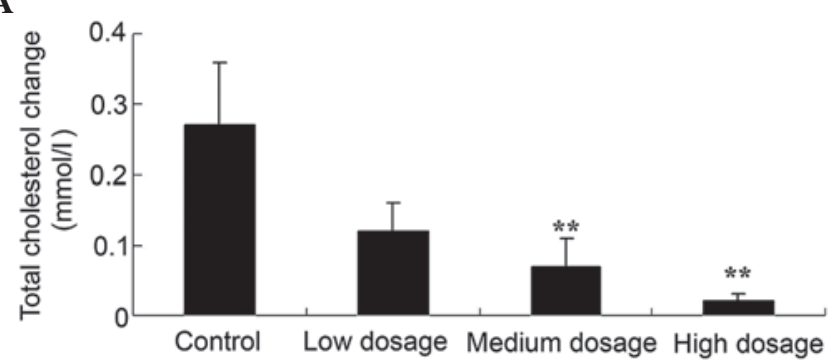

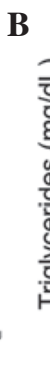

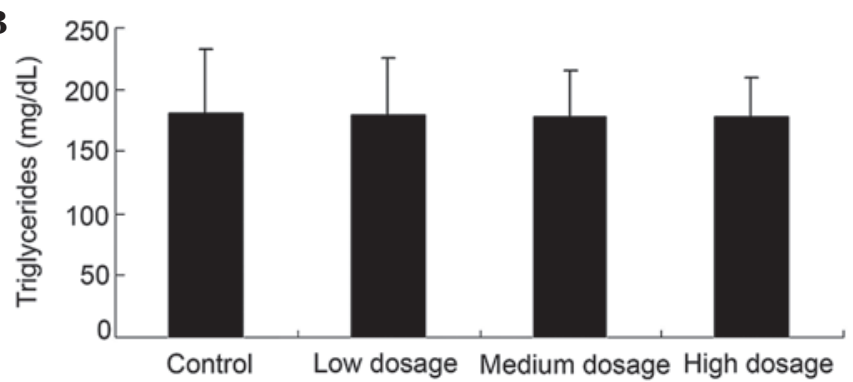

Figure 3. Effect of different doses of vitamin D supplementation on lipid concentrations, including (A) total cholesterol change and (B) triglyceride levels, in patients with gestational diabetes mellitus. Data are presented as mean \pm standard deviation. ${ }^{* *} \mathrm{P}<0.01$ vs. control group.

groups displayed significantly decreased total cholesterol change in pregnant women with GDM, as compared with the control $(0.07$ and 0.02 vs. $0.27 \mathrm{mmol} / 1 ; \mathrm{P}<0.01)$. By contrast, the levels of triglycerides in the low, medium and high vitamin D dosage groups (179, 178 and $178 \mathrm{mg} / \mathrm{dl}$, respectively) were not significantly different, as compared with the control group (180 mg/dl) (Fig. 3B; P>0.05).

Vitamin D supplementation does not reduce inflammation in patients with GDM. Fig. 4 revealed that low, medium and 


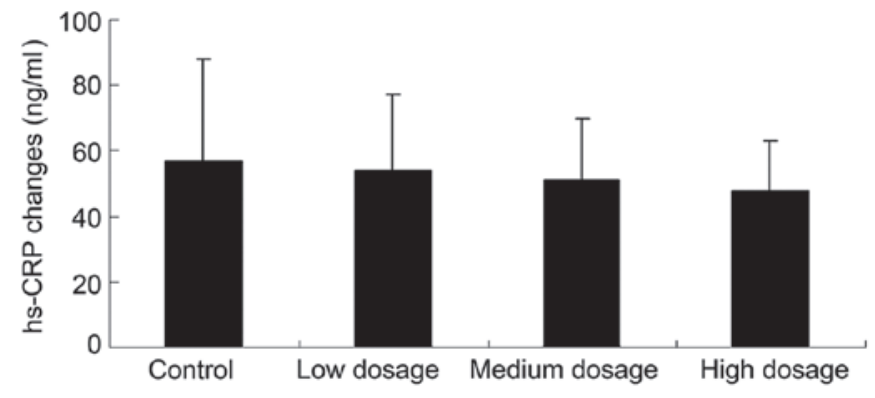

Figure 4. Effect of different doses of vitamin D supplementation on inflammation, as measured by hs-CRP concentration, in patients with gestational diabetes mellitus. Data are presented as mean \pm standard deviation. hs-CRP, high-sensitivity c-reactive protein.
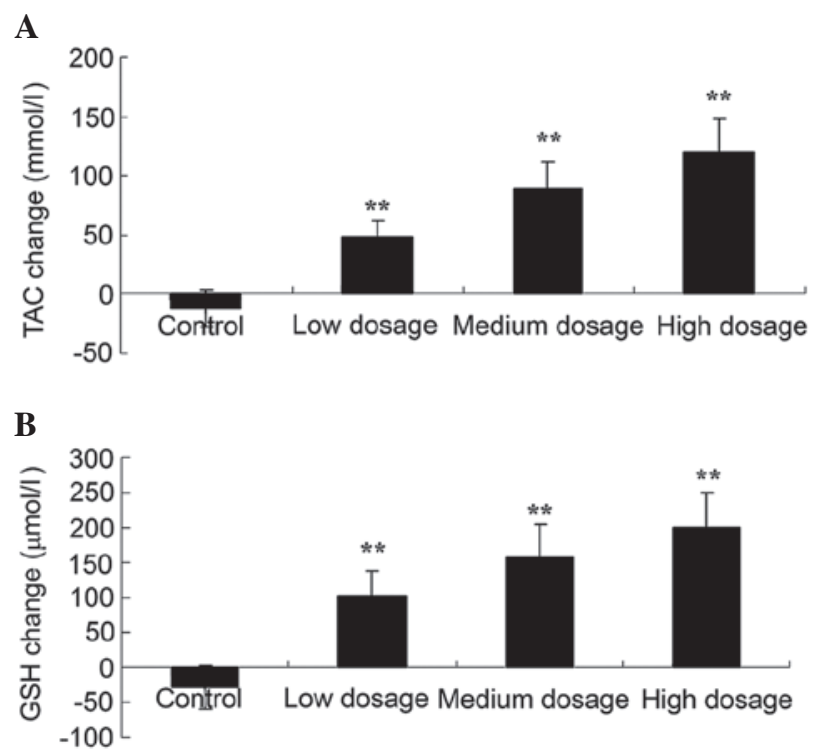

Figure 5. Effect of different doses of vitamin D supplementation on oxidative stress, as assessed by (A) TAC and (B) GSH levels, in patients with gestational diabetes mellitus. Data are presented as mean \pm standard deviation. ${ }^{* *} \mathrm{P}<0.01$ vs. control group. TAC, total antioxidant capacity; GSH, total glutathione.

high dose vitamin D supplementation (54, 51 and $48 \mathrm{ng} / \mathrm{ml}$, respectively) does not significantly reduce the hs-CRP levels of patients with GDM, as compared with the control group (57 ng/ml; P>0.05).

Vitamin D supplementation increases TAC and total GSH in patients with GDM. Fig. 5 revealed that high, medium and low doses of vitamin D supplementation all significantly increased TAC (120, 89 and $49 \mathrm{mmol} / \mathrm{l}$, respectively; Fig. 5A) and total GSH (199, 158 and $102 \mu \mathrm{mol} / 1$, respectively; Fig. 5B) levels in patients with GDM, as compared with the control groups (12 $\mathrm{mmol} / 1$ and $-28 \mu \mathrm{mol} / 1$, respectively; $\mathrm{P}<0.01$ ).

\section{Discussion}

Medical nutrition therapy (MNT) is the foundation of treating diabetes and is an essential measure for the prevention of phase and control phase in the natural course of diabetes. In 2002, the American Diabetes Association (ADA) proposed the evidence-based diabetes nutrition supply standard and and established a classification standard for scientific evidence (20). ADA indicated that patients with GDM who adhered to personalized nutrition therapy from a registered dietitian were more likely to achieve treatment goals. The pathophysiological features of GDM are unique and require close management; if poorly managed, maternal hypoglycemia, ketoacidosis and high blood sugar, in addition to other complications, may occur (21). The high blood sugar levels often observed in patients with GDM can lead to fetal hyperglycemia, while GDM-induced hyperosmolarity is typically treated with diuretics, resulting in increased urination; consequently, sugar levels are high in the amniotic fluid, which in turn stimulates the secretion of amniotic membrane and may eventually lead to excessive amniotic fluid (22). Additionally, hyperglycemia stimulates fetal insulin secretion. Excessive insulin levels may reduce fetus alveolar surface material, resulting in delayed fetal lung maturity and therefore increasing the incidence of neonatal respiratory distress syndrome (23). Furthermore, hyperinsulinemia persists in the newborn following birth and removal from the environment of the mother's hyperglycemia; thus, the newborn is prone to neonatal hypoglycemia (24). The present study involved a randomized, double-blind, placebo-controlled clinical trial conducted on 133 patients with a GDM diagnosis of $<12$ weeks. The general characteristics and dietary intakes of patients with GDM in each group of the trial were similar, which demonstrated that these patient charcteristics did not differ prior to intervention.

In pregnant women with elevated blood sugar levels, fetuses may experience fetal hyperglycemia, leading to fetal islet cell proliferation, increased insulin secretion and promotion of the synthesis of protein and fat, in addition to the inhibition of lipolysis, thus leading to the occurrence of fetal macrosomia (25). GDM insulin resistance and hyperinsulinemia may cause microvascular disease, resulting in the thickening of the basement membrane in capillary walls. This also contributes to the occurrence of maternal hypertensive disorders in pregnancy (26). In the present study, patients in the groups receiving high and medium doses of vitamin D supplementation were observed to display reduced insulin, HOMA-IR and total cholesterol levels. These results demonstrated that high-dose vitamin D supplementation significantly improved insulin resistance in pregnant women with GDM. However, the levels of FPG and triglycerides exhibited no significant differences in the groups receiving vitamin D supplementation compared with the control group. Senti et al reported that vitamin D supplementation was a potential target for patients with GDM (27). Yap et al also revealed that the effect of vitamin D supplementation (5,000 IU per day) prevented glucose metabolism during pregnancy (28).

Diabetes is a vascular disease, as well as a disease of hyperglycemia. In addition, diabetes may also be an inflammatory disease (29). Inflammation, immune system activation and the corresponding metabolic changes in an organism are subject to the regulation of the nervous and endocrine systems, which affect organism function through feedback pathways, forming complex nerve-endocrine-immune system networks (30). GDM and obesity involve insulin resistance as a common feature in their disease pathogenesis. A variety of inflammatory cytokines generated by the activation of the immune 
system, including tumor necrosis factor- $\alpha$, interleukin- 6 and CRP, can induce insulin resistance (31). In the present study, hs-CRP levels in patients with GDM were not affected by high dosage vitamin D supplementation. Similarly, Asemi et al revealed that vitamin D supplementation does not affect inflammation in pregnant women with GDM $(17,32)$.

The oxidative stress imbalance involves excessive oxidative generation and inadequate levels of antioxidants. It has been revealed that oxidative stress imbalance is important in the pathophysiological processes involved in vascular diseases, including diabetes, hypertension and atherosclerosis (33). An oxidative stress imbalance is also present in GDM patients and can cause vascular injury and adverse pregnancy events. Multiple adverse pregnancy outcomes are associated with GDM, including fetal growth restriction, preterm birth, hypertensive disorders of pregnancy, stillbirth and miscarriage, all of which are associated with abnormal placental circulation (34). The present study demonstrated that high, medium and low dosages of vitamin D supplementation resulted in dose-dependent increases in total TAC and GSH levels in patients with GDM, which suggested that high-dose vitamin D supplementation provided the greatest anti-oxidation effects. Asemi et al previously presented data in support of this, revealing that vitamin D supplementation significantly increased malondialdehyde and GSH levels (35).

In conclusion, high-dose vitamin D supplementation (50,000 IU) every 2 weeks significantly decreased levels of insulin resistance in pregnant women with GDM. However, it did not affect FPG, triglyceride or inflammation levels. The results of the present study suggest that high-dose vitamin D supplementation $(50,000 \mathrm{IU}$ every 2 weeks) is recommended for pregnant women with GDM from the 12th week of pregnancy until delivery.

\section{Acknowledgements}

The present study was supported by the Research Project of Shanghai City (grant no. 14411965600) and The National Natural Science Fund (grant no. 81471469).

\section{References}

1. Zhao C, Zhang T, Shi Z, Ding H and Ling X: MicroRNA-518d regulates PPAR $\alpha$ protein expression in the placentas of females with gestational diabetes mellitus. Mol Med Rep 9: 2085-2090, 2014.

2. Asemi Z, Karamali M, Jamilian M, Foroozanfard F, Bahmani F, Heidarzadeh Z, Benisi-Kohansal S, Surkan PJ and Esmaillzadeh A6: Magnesium supplementation affects metabolic status and pregnancy outcomes in gestational diabetes: A randomized, double-blind, placebo-controlled trial. Am J Clin Nutr 102: 222-229, 2015.

3. AsemiZ, Tabassi Z, Samimi M,Fahiminejad T and Esmaillzadeh A: Favourable effects of the dietary approaches to stop hypertension diet on glucose tolerance and lipid profiles in gestational diabetes: A randomised clinical trial. Br J Nutr 109: 2024-2030, 2013.

4. Xin G, Du J, Wang YT and Liang TT: Effect of oxidative stress on heme oxygenase-1 expression in patients with gestational diabetes mellitus. Exp Ther Med 7: 478-482, 2014.

5. Aittasalo M, Raitanen J, Kinnunen TI, Ojala K, Kolu P and Luoto R: Is intensive counseling in maternity care feasible and effective in promoting physical activity among women at risk for gestational diabetes? Secondary analysis of a cluster randomized NELLI study in Finland. Int J Behav Nutr Phys Act 9: 104, 2012.

6. Jao J, Wong M, Van Dyke RB, Geffner M, Nshom E, Palmer D, Muffih PT, Abrams EJ, Sperling RS and Leroith D: Gestational diabetes mellitus in HIV-infected and -uninfected pregnant women in Cameroon. Diabetes Care 36: e141-142, 2013.
7. Han S, Middleton PF, Bubner TK and Crowther CA: Women's views on their diagnosis and management for borderline gestational diabetes mellitus. J Diabetes Res: 209215, 2015.

8. Korpi-Hyövälti E, Heinonen S, Schwab U, Laaksonen DE and Niskanen L: Effect of intensive counselling on physical activity in pregnant women at high risk for gestational diabetes mellitus. A clinical study in primary care. Prim Care Diabetes 6: 261-268, 2012.

9. Soydinc HE, Davutoglu V, Sak ME, Ercan S, Evsen MS, Kaya H, Oylumlu M, Buyukaslan H and Sari I: Circadian variation of blood pressure is impaired in normotensive pregnant women with gestational diabetes mellitus. Clin Exp Hypertens 35: 128-133, 2013.

10. Lohse N, Marseille E and Kahn JG: Development of a model to assess the cost-effectiveness of gestational diabetes mellitus screening and lifestyle change for the prevention of type 2 diabetes mellitus. Int J Gynaecol Obstet 115 (Suppl 1): S20-S25, 2011.

11. Wojcik M, Zieleniak A, Zurawska-Klis M, Cypryk K and Wozniak LA: Increased expression of immune-related genes in leukocytes of patients with diagnosed gestational diabetes mellitus (GDM). Exp Biol Med (Maywood) 2015.

12. Nobles C, Marcus BH, Stanek EJ III, Braun B, Whitcomb BW, Solomon CG, Manson JE, Markenson G and Chasan-Taber L: Effect of an exercise intervention on gestational diabetes mellitus: A randomized controlled trial. Obstet Gynecol 125: 1195-1204, 2015

13. Djelti F, Merzouk H, Merzouk SA and Narce M: In vitro effects of oil's fatty acids on $\mathrm{T}$ cell function in gestational diabetic pregnant women and their newborns. J Diabetes 7: 512-522, 2015.

14. Driscoll KA and Young-Hyman D: Use of technology when assessing adherence to diabetes self-management behaviors. Curr Diab Rep 14: 521, 2014.

15. Ji LL, Tong L, Peng JB, Jin XH, Wei D, Xu BK and Wang ZY: Changes in the expression of the vitamin $D$ receptor and LVSCC-A1C in the rat hippocampus submitted to single prolonged stress. Mol Med Rep 9: 1165-1170, 2014.

16. Burris $\mathrm{HH}$ and Camargo CA Jr: Time for large randomised trials of vitamin D for women with gestational diabetes mellitus to improve perinatal health outcomes. Diabetologia 57: 1746-1748, 2014.

17. AsemiZ, Hashemi T,Karamali M,Samimi Mand Esmaillzadeh A: Effects of vitamin D supplementation on glucose metabolism, lipid concentrations, inflammation, and oxidative stress in gestational diabetes: A double-blind randomized controlled clinical trial. Am J Clin Nutr 98: 1425-1432, 2013.

18. Mozaffari-Khosravi H, Hosseinzadeh-Shamsi-Anar M, Salami MA, Hadinedoushan H and Mozayan MR: Effects of a single post-partum injection of a high dose of vitamin D on glucose tolerance and insulin resistance in mothers with first-time gestational diabetes mellitus. Diabet Med 29: 36-42, 2012.

19. Hossain N, Kanani FH, Ramzan S, Kausar R, Ayaz S, Khanani R and Pal L: Obstetric and neonatal outcomes of maternal vitamin D supplementation: Results of an open-label, randomized controlled trial of antenatal vitamin D supplementation in Pakistani women. J Clin Endocrinol Metab 99: 2448-2455, 2014.

20. Koloverou E, Panagiotakos DB, Pitsavos C, Chrysohoou C, Georgousopoulou EN, Laskaris A and Stefanadis C; ATTICA Study group: The evaluation of inflammatory and oxidative stress biomarkers on coffee-diabetes association: results from the 10-year follow-up of the ATTICA Study (2002-2012). Eur J Clin Nutr 69: 1220-1225, 2015.

21. Docherty LE, Kabwama S, Lehmann A, Hawke E, Harrison L, Flanagan SE, Ellard S, Hattersley AT, Shield JP, Ennis S et al: Clinical presentation of $6 \mathrm{q} 24$ transient neonatal diabetes mellitus (6q24 TNDM) and genotype-phenotype correlation in an international cohort of patients. Diabetologia 56: 758-762, 2013.

22. Xie BG, Jin S and Zhu WJ: Expression of toll-like receptor 4 in maternal monocytes of patients with gestational diabetes mellitus. Exp Ther Med 7: 236-240, 2014.

23. Livingston EG, Herbert WN, Hage ML, Chapman JF and Stubbs TM: Use of the TDx-FLM assay in evaluating fetal lung maturity in an insulin-dependent diabetic population. The Diabetes and Fetal Maturity Study Group. Obstet Gynecol 86: 826-829, 1995.

24. Yilmaz Ö, Küçük M, Kebapçilar L, Altindag T, Yüksel A, YuvançHO,DalT and Savran Y: Macrophage migration-inhibitory factor is elevated in pregnant women with gestational diabetes mellitus. Gynecol Endocrinol 28: 76-79, 2012.

25. Savvidou MD, Akolekar R, Samaha RB, Masconi AP and Nicolaides KH: Maternal serum 25-hydroxyvitamin D levels at 11(+0) -13(+6) weeks in pregnant women with diabetes mellitus and in those with macrosomic neonates. BJOG 118: 951-955, 2011. 
26. Goker Tasdemir U, Tasdemir N, Kilic S, Abali R, Celik C and Gulerman HC: Alterations of ionized and total magnesium levels in pregnant women with gestational diabetes mellitus. Gynecol Obstet Invest 79: 19-24, 2015.

27. Senti J, Thiele DK and Anderson CM: Maternal vitamin D status as a critical determinant in gestational diabetes. J Obstet Gynecol Neonatal Nurs 41: 328-338, 2012.

28. Yap C, Cheung NW, Gunton JE, Athayde N, Munns CF, Duke A and McLean M: Vitamin D supplementation and the effects on glucose metabolism during pregnancy: A randomized controlled trial. Diabetes Care 37: 1837-1844, 2014.

29. Taha H, Arya A, Paydar M, Looi CY, Wong WF, Vasudeva Murthy CR, Noordin MI, Ali HM, Mustafa AM and Hadi AH: Upregulation of insulin secretion and downregulation of pro-inflammatory cytokines, oxidative stress and hyperglycemia in STZ-nicotinamide-induced type 2 diabetic rats by Pseuduvaria monticola bark extract. Food Chem Toxicol 66: 295-306, 2014.

30. Yu J, Zhou Y, Gui J, Li AZ, Su XL and Feng L: Assessment of the number and function of macrophages in the placenta of gestational diabetes mellitus patients. J Huazhong Univ Sci Technolog Med Sci 33: 725-729, 2013.
31. Derbent AU, Simavli SA, Kaygusuz I, Gumus II, Y1lmaz S, Yildirim M and Uysal S: Serum hepcidin is associated with parameters of glucose metabolism in women with gestational diabetes mellitus. J Matern Fetal Neonatal Med 26: 1112-1115, 2013.

32. Asemi Z, Karamali $M$ and Esmaillzadeh A: Effects of calcium-vitamin D co-supplementation on glycaemic control, inflammation and oxidative stress in gestational diabetes: A randomised placebo-controlled trial. Diabetologia 57: 1798-1806, 2014.

33. Vural M, Camuzcuoglu H, Toy H, Cece H, Aydin H, Eren MA, Kocyigit A and Aksoy N: Evaluation of the future atherosclerotic heart disease with oxidative stress and carotid artery intima media thickness in gestational diabetes mellitus. Endocr Res 37: 145-153, 2012.

34. López-Tinoco C, Roca M, García-Valero A, Murri M, Tinahones FJ, Segundo C, Bartha JL and Aguilar-Diosdado M: Oxidative stress and antioxidant status in patients with late-onset gestational diabetes mellitus. Acta Diabetol 50: 201-208, 2013.

35. Asemi Z, Samimi M, Tabassi Z, Shakeri H and Esmaillzadeh A: Vitamin D supplementation affects serum high-sensitivity C-reactive protein, insulin resistance, and biomarkers of oxidative stress in pregnant women. J Nutr 143: 1432-1438, 2013. 